\title{
A Hop by Hop Congestion Control Protocol to Mitigate Traffic Contention in Wireless Sensor Networks
}

\author{
Kamal Kumar Sharma, Dr. Harbhajan Singh and Dr. R.B Patel
}

\begin{abstract}
In wireless sensor networks, contention causes message collisions, which are very likely to occur when traffic is frequent and correlated, and hence decreases the lifetime of the network. Also congestion happens due to contention which is caused by concurrent transmissions. In this paper, we propose to develop a Hop-by-hop congestion control protocol for wireless sensor networks to mitigate traffic contention. In our proposed protocol, each node calculates its node rank based on the parameters buffer Size, hop count, channel busy ratio and MAC overhead. When the node rank crosses a threshold value $T$, the sensor node will set a congestion bit in every packet it forwards. If the congestion bit is set, the downstream node calculates the Rate Adjustment Feedback based on the rank and propagates this value upstream towards the source nodes. The source nodes will adjust their transmission rates dynamically based on this feedback. By simulation results, we show that our proposed protocol attains better throughput and packet delivery ratio with reduced energy consumption.
\end{abstract}

Index Terms-Wireless Sensor Networks, Traffic Contention, Congestion Control Protocol, MAC, Rate Adjustment Feedback.

\section{INTRODUCTION}

In recent years, in order to access the network resources easily and efficiently, there are several network protocols for users with portable devices. Wireless Sensor Network (WSN) is considered as one of the growing technologies. The sensor nodes are usually scattered and it is not necessary to predetermine the position of the sensor nodes in the sensor networks. It can also be expressed that a WSN is a self-configuring network of tiny nodes connected by wireless links and communicates with a sink node. Moreover, WSN has limited bandwidth and limited battery power, due to the utilization of wireless channel [1]. Wireless Sensor networks (WSNs) are used for an extensive range of applications in the military, health, environment, agriculture and smart office domain.

When two nearby sensor nodes attempt to access the communication channel simultaneously, contention takes place. If a MAC protocol does not allow any collisions, then it is considered as contention-free. The existing contentionfree MAC protocols (eg. TDMA) assume that the sensor nodes are time-synchronized, which is not possible for the large scale sensor networks [3].

Kamal Kumar Sharma, Associate Professor, Shree Ram Institute of Engineering \& Technology, Yamuna Nagar, Haryana (kamalkumarsharmaphd@gmail.com)

Dr. Harbhajan Singh, Professor, RIEIT, Railmajra, Punjab

Dr. R.B Patel, Professor, MITS, Rajasthan
MAC protocols are either contention-based or contentionfree. Contention-based MAC protocols are also known as random access protocols, requiring no coordination among the nodes accessing the channel [3]. Some of the contention based protocols are CSMA, IEEE 802.11, TMAC, and SMAC [4].

In the contention-free MAC protocols, the nodes are following some particular schedule which guarantees collision-free transmission times. Typical examples of contention-free protocols are: Frequency Division Multiple Access (FDMA); Time Division Multiple Access (TDMA); Code Division Multiple Access (CDMA) [3].

Congestion happens due to contention caused by concurrent transmissions [2]. Contention causes message collisions, which are very likely to occur when traffic is frequent and correlated, and it decreases the lifetime of a sensor network [3].

The contention based protocols are not suitable for sensor networks because, when the traffic is frequent or correlated, the performance of those protocols is weak and experiences stability problems [3].

Listening before transmitting is the main idea of the contention based protocols. When these protocols continuously sense the carrier, it results in energy wastage on idle listening [4].

Several types of traffic from simple periodic reports to unpredictable bursts of messages activated by sensed events are delivered by WSN [2]. Congestion is a likely event due to time varying wireless channel condition and contention which are caused as a result of interference by concurrent transmissions, even for the simple network topology and periodic traffic. Congestion obstructs reliable event perception, since it causes huge packet loss [2].

The data transmission is opportunistic in sensor network. The data gets transmitted whenever the nodes within the event radius receive the channel access. Therefore, congestion occurs when multiple nodes within the event radius contend for the channel simultaneously. One of the major reasons for congestion in sensor network is the packet loss due to collision. Moreover, many-to-one generalization is followed by the data transmission in sensor network under single or multiple sink situations. Therefore, communicating more data than the other nodes is responsible by irrespective number of events and their location nodes nearer to the sink or in the intersection of multiple routes [2].

The following are the drawbacks which are caused due to congestion [2]:

- Increases energy dissipation rates of sensor nodes 
- Causes a lot of packet loss, which in turn diminish the network throughput and

- Causes delays in fair event detections and reliable data transmission.

In wireless sensor networks, congestion causes overall channel quality to degrade and loss rates to rise, leads to buffer drops and increased delays (as in wired networks), and tends to be grossly unfair toward nodes whose data has to traverse a larger number of radio hops [6].

The good congestion control measures for the wired networks are the packet loss, queue length and link utilization. But mainly for two reasons, these measures cannot be applied directly to the sensor networks. First, due to the medium contention, the occurrence of packet loss and large queue length may indicate that severe congestion has occurred already. Therefore it does not provide any time for the network to react quickly. Second, a wireless link is shared by all the neighboring nodes unlike a wired link which is normally between the two nodes. Therefore, it is complex to trace the changes in the status of the wireless link which makes difficult to provide accurate control.

A candidate of congestion signal should reflect the condition of MAC contention and collision because MAC contention is tightly coupled with congestion. The candidate should indicate the available buffer size to make use of the shared channel completely without causing severe congestion and packet collision.

In this paper, we propose a Hop-by-hop congestion control protocol for wireless sensor networks to mitigate traffic contention.

\section{RELATED WORK}

Young-Duk Kim et al [1] have proposed a Distance Adaptive Contention Window (DACW) modified IEEE 802.15.4 standard. The key mechanism of DCAW is a dynamical channel access MAC protocol, which is to adjust Contention Window (CW) according to the hop count distance to sink and traffic condition. With DACW, each sensor node can achieve self-routing capability with low overhead and performance enhancement.

Md. Mamun-Or-Rashid et al [2] have proposed an energy efficient congestion avoidance protocol that includes source count based hierarchical and load adaptive medium access control. Their proposed mechanism ensures load adaptive media access to the nodes and thus achieves fairness in event detection. Their results shows that their scheme exhibits more than $90 \%$ delivery ratio with retry limit 1 , even under bursty traffic condition which is good enough for reliable event perception.

Ian F. Akyildiz et al [7] have developed a unified crosslayer protocol which replaces the entire traditional layered protocol architecture that has been used so far in WSNs. The objective of their proposed cross layer protocol is highly reliable communication with minimal energy consumption, adaptive communication decisions and local congestion avoidance. Their protocol operation is governed by the new concept of initiative determination. Based on this concept, the cross-layer protocol performs received based contention, local congestion control, and distributed duty cycle operation in order to realize efficient and reliable communication in WSN.

Wafa Ben Jaballah et al [8] have enhanced the QoS in sensor networks. They have presented an approach which takes advantage of the standard 802.11e EDCA protocol [16] that ensures effective end to end delay and good quality of traffic. They have tried to improve the provision of quality of service in sensor networks by offering a new approach which aims to improve the mechanism of service differentiation implemented in the 802.11e. But it is suitable only for multimedia flows using 802.11e MAC protocol.

Hongwei Zhang et al [9] have designed a window-less block acknowledgment scheme to improve channel utilization and to reduce ack-loss that guarantees continuous packet forwarding and replicates the acknowledgment for a packet. They have introduced a differentiated contention control to alleviate retransmission-incurred channel contention. Moreover, they have designed mechanisms to handle varying ack-delay and to reduce delay in timer based retransmissions.

Ajit Warrier et al [10] have presented a new hybrid MAC scheme - Z-MAC, for sensor networks. Z-MAC is robust topology changes and clock synchronization errors; in the worst case its performance falls back to that of CSMA. They have implemented Z-MAC in Tiny OS and evaluated its channel utilization, energy, latency and fairness over singlehop, two hop and multi-hop sensor network topologies constructed using Mica2. Their results have shown that ZMAC has remarkably better data throughput than existing sensor MAC protocols while consuming comparable energy.

Muhammad Mostafa Monowar et al [12] have proposed an efficient scheme to control multi-path congestion so that the sink can get priority based throughput for heterogeneous data. They have used packet service ratio for detecting congestion as well as performed hop-by-hop multi-path congestion control based on that metric. Their simulation results have demonstrated the effectiveness of their proposed approach.

Chonggang Wang et al [13] have proposed a node priority-based congestion control protocol (PCCP) for wireless sensor networks. In PCCP, node priority index has been introduced to reflect the importance of each node. PCCP uses packet inter-arrival time along with packet service time to measure a parameter defined as congestion degree and furthermore imposes hop-by-hop control based on the measured congestion degree as well as the node priority index. PCCP controls congestion faster and more energy-efficiently than other known techniques.

\section{SySTEM DESIGN AND PRotocol OVERVIEW}

In our proposed protocol, each node calculates its node rank (NR) based on the parameters Buffer Size, Hop count, Channel Busy Ratio and MAC overhead.

Here the channel busyness ratio [11] represents the interference level and is defined as the ratio of time intervals when the channel is busy due to successful transmission or collision to the total time. After estimating its rank, a node forwards this to its downstream node. When NR crosses a 
threshold $\mathrm{T}$, then the node will set the congestion bit (CB) in every packet it forwards.

On receiving this node rank, the downstream node will first check for the congestion bit. If it is not set, it will simply computes its node rank and adds it to the rank obtained from its previous node and passes on to the next node. On the other hand, if the congestion bit is set, the node calculates the Rate Adjustment Feedback (RAF) based on the rank and transmits it towards the source as a feedback. On receiving this feedback, the source nodes will adjust their transmission rate and retransmit the data again.

\section{A. MAC Overhead}

From the MAC layer, contention and channel interference of the nodes can be estimated and notified to the application layer. Based on these estimations, at the application layer, the transmission rate can be adjusted, to avoid congestion.

In this network, we consider IEEE 802.11 MAC with the distributed coordination function (DCF). It has the packet sequence as request-to-send (RTS), clear-to-send (CTS), and data, acknowledge (ACK). The amount of time between the receipt of one packet and the transmission of the next is called a short inter frame space (SIFS). Then the channel occupation due to MAC contention will be

$$
C_{\text {occ }}=t_{R T S}+t_{C T S}+3 t_{\text {SIFS }}
$$

Where $t_{R T S}$ and $t_{C T S}$ are the time consumed on $R T S$ and CTS , respectively and $t_{S I F S}$ is the SIFS period..

Then the $M A C$ overhead $O H_{M A C}$ can be represented as

$$
O H_{M A C}=C_{o c c}+t_{a c c}
$$

Where $t_{a c c}$ is the time taken due to access contention.

The amount of $M A C$ overhead is mainly dependent upon the medium access contention, and the number of packet collisions. That is, $O H_{M A C}$ is strongly related to the congestion around a given node.

\section{B. Channel Busy Ratio}

Channel Busy Ratio (CHBR) represents the interference level and is given by

$$
C H B R=\frac{B I n t}{\text { Ttot }}
$$

Where BInt represents the time intervals when the channel is busy due to successful transmission or collision and Ttot represents the total time.

\section{HOP BY HOP CONGESTION CONTROL}

Each node calculates its node rank NR based on the following parameters $B$ Size , $H C, C B H R$ and $\mathrm{OH}_{\mathrm{MAC}}$, where $B$ Size is the buffer size of the node and $H C$ is the hop count value.

$$
N R=\alpha 1 . B_{S i z e}+\alpha 2 \cdot H+\alpha 3 \cdot C H B R+\alpha 4 O H_{M A C}
$$

Here $\alpha 1, \alpha 2, \alpha 3$ and $\alpha 4$ are constant weight factors whose values between 0 to 1 .

Each data packet has a congestion bit (CB) in its header. Every sensor node maintains a. threshold T. When NR crosses the value of $\mathrm{T}$ the node will set its $\mathrm{CB}$ in every packet it forwards.
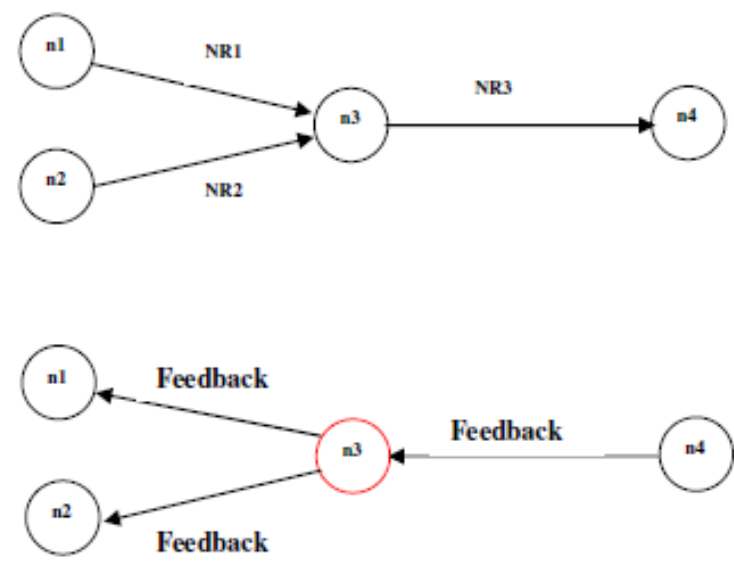

Fig.1 a) Node Rank Propagation b) Feedback propagation

\section{Algorithm}

1. Nodes $\mathrm{n} 1$ and $\mathrm{n} 2$ estimate their rank $\mathrm{NR}_{1}$ and $\mathrm{NR}_{2}$ and forwards this to its downstream node $\mathrm{n} 3$ along with the data packet.

2. On receiving $N_{1}$ and $N R 2, n 3$ will first check the $\mathrm{CB}$ value for both. If it is not set, it will simply computes its node rank NR3 and adds it to the rank obtained from nodes $\mathrm{n} 1$ and $\mathrm{n} 2$ and then passes on to the next node n4. Figure 1.a illustrates this process.

3. On the other hand, if the $\mathrm{CB}$ is set, the node $\mathrm{n} 3$ calculates the Rate Adjustment Feedback (RAF) based on the rank as

$$
R A F=\left(A_{\text {rate }} / H C\right)-\Sigma O H_{M A C i}-\Sigma C B H R_{i}
$$

Where $A_{\text {Rate }}$ is the arrival rate of packets at node n2, which is given by,

$$
A_{\text {Rate }}=N P / t
$$

Here NP - is the number of packets received and $t$ is the time of interval for the

packet transmission.

4. Then, this RAF is propagated upstream towards the source nodes $\mathrm{n} 1$ and $\mathrm{n} 2$, as a feedback packet. Figure 1.b illustrates this process.

5. On receiving this feedback packet, the upstream nodes will adjust their transmission rate Nrate dynamically as follows

$$
\text { Nrate }=\text { Nrate }-R A F
$$

Thus the traffic rate is adaptively adjusted according to the MAC contention and buffer size.

6. The procedure is repeated for the all the hops towards the sink. 


\section{EXPERIMENTAL RESULTS}

\section{A. Simulation Model and Parameters}

We use NS2 [14] to simulate our proposed protocol. In our simulation, the channel capacity of mobile hosts is set to the same value: $2 \mathrm{Mbps}$. We use the distributed coordination function (DCF) of IEEE 802.11 as the MAC layer protocol.

In our simulation, sensor nodes of sizes 25, 50, 75 and 100 are deployed in a $1000 \mathrm{mr} \times 1000 \mathrm{~m}$ region for 50 seconds simulation time. All nodes have the same transmission range of 250 meters. The simulated traffic is Constant Bit Rate (CBR). The simulation settings are summarized in the following table.(Table 1).

TABLE1: SIMULATION SETTINGS
\begin{tabular}{|l|l|}
\hline No. of Nodes & $25,50,75$ and 100 \\
\hline Area Size & $1000 \mathrm{X} 1000$ \\
\hline Mac & 802.11 \\
\hline Simulation Time & $50 \mathrm{sec}$ \\
\hline Traffic Source & CBR \\
\hline Packet Size & 512 \\
\hline Transmit Power & $0.360 \mathrm{w}$ \\
\hline Receiving Power & $0.395 \mathrm{w}$ \\
\hline Idle Power & $0.335 \mathrm{w}$ \\
\hline Initial Energy & $5.1 \mathrm{~J}$ \\
\hline No. of sources & 5 \\
\hline Transmission Rate & $250,500,750$ and $1000 \mathrm{~kb}$. \\
\hline$\alpha 1$ & 0.2 \\
\hline$\alpha 2$ & 0.3 \\
\hline$\alpha 3$ & 0.3 \\
\hline$\alpha 4$ & 0.2 \\
\hline
\end{tabular}

\section{B. Performance Metrics}

We compare the performance of our proposed HHCC protocol with Dynamic Contention Window based Congestion Control (DCWCC) [2] protocol. We evaluate mainly the performance according to the following metrics:

Aggregated Throughput: We measure aggregated throughput in terms of no. packets received.

Average Energy Consumption: The average energy consumed by the nodes in receiving and sending the packets are measured.

Packet Delivery Ratio: It is the ratio of the fraction of packets received successfully and the total no. of packets sent.

The performance results are presented graphically in the next section.

\section{Results}

\section{1) Varying No. Of Nodes}

In order to test the scalability, in the first experiment, we measure the performance of the protocols by varying the no. of nodes as $25,50,75$ and 100 .

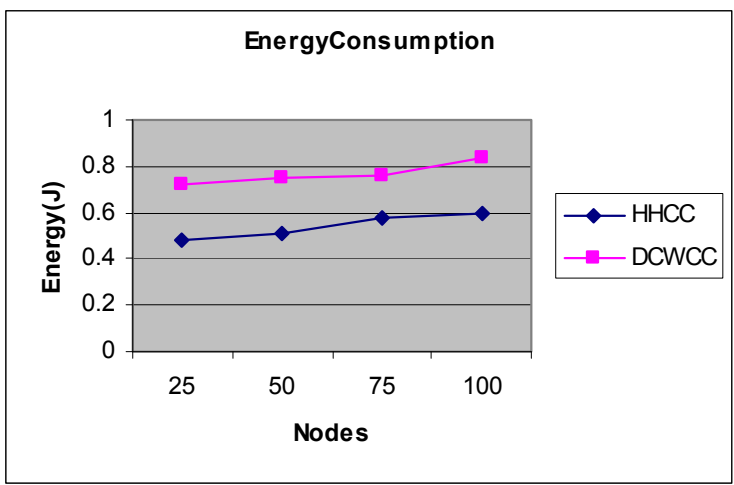

Fig 2. Energy Consumption Vs No. of Nodes

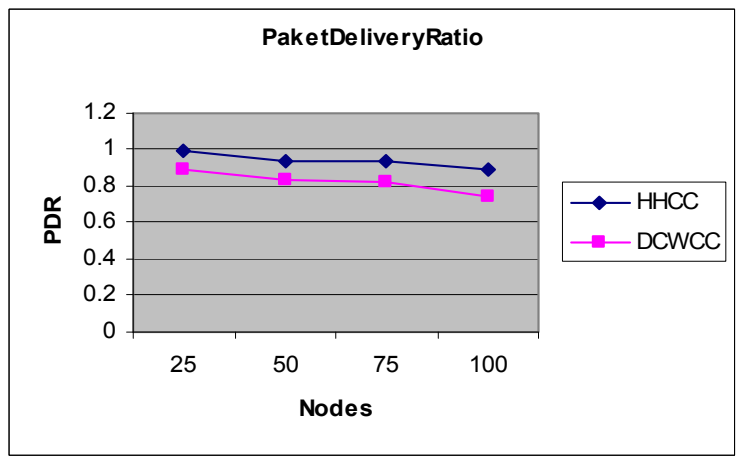

Fig 3. Packet Delivery Ratio Vs No. of Nodes

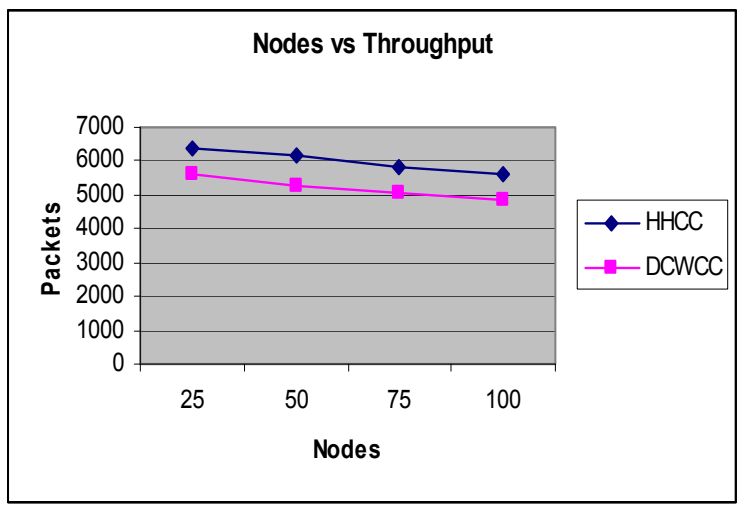

Fig 4. Throughput Vs No. of Nodes

Fig. 2 shows that the average energy consumed by the nodes in receiving and sending the data. We can see from the figure, the average energy consumption of the nodes increases as the number of nodes increases from 15 to 100 . Since HHCC make use hop by hop congestion control, the overall energy consumption values are considerably less in HHCC when compared to the DCWCC protocol.

Fig. 3 presents the packet delivery Ratio (PDR) values for both the protocols. As the number of nodes increases, the number of hops involved in routing the data towards the sink, also increases. So the PDR values are slightly decreased. But we can see that the PDR for HHCC is more when compared to the DCWCC protocol, as the sending rate is immediately controlled by the sources, when congestion occurs.

Fig.4 shows the throughput obtained with for both the protocols when the number of nodes is increased. For the same reason stated above, the throughput of HHCC is significantly more than the DCWCC, as the number of nodes increases. 


\section{2) Varying the Transmission Rate}

To see the effect of increased sending rate, in the second experiment, we measure the performance of the protocols by varying the transmission rate as $250,500,750$ and $1000 \mathrm{~Kb}$.

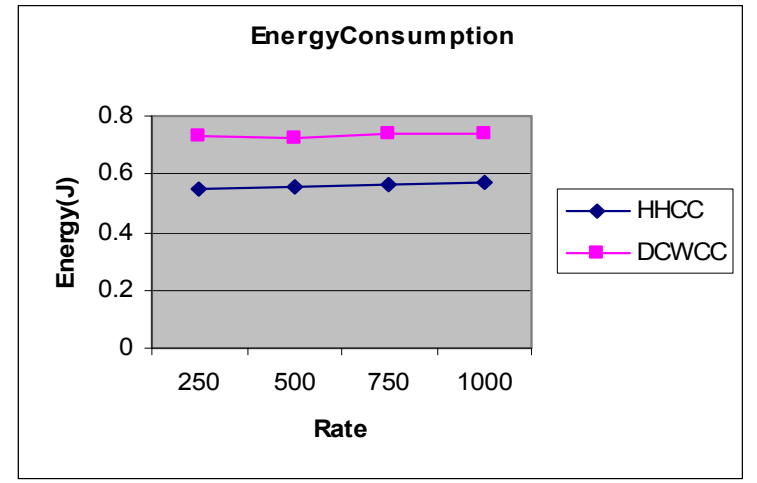

Fig 5.Energy Consumption Vs Rate

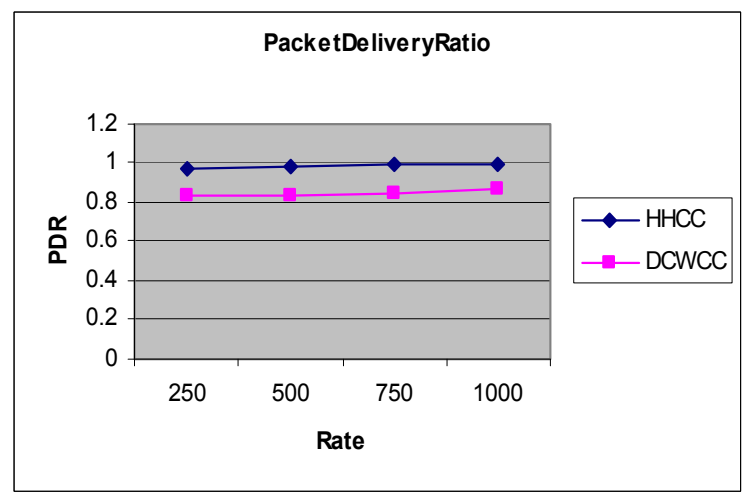

Fig 6. Packet Delivery ratio Vs Rate

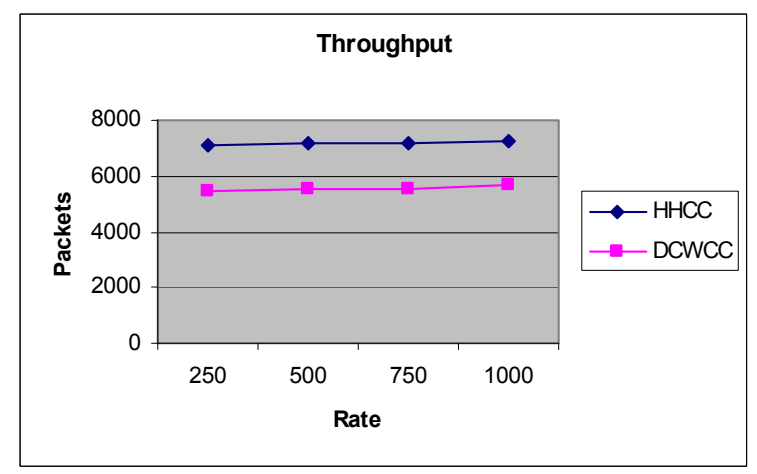

Fig 7. Throughput Vs Rate

Fig 5 shows that the average energy consumption of both the protocols, when the sending rate of the sources increases. When the sending rate is increased, it results in more energy consumption of the nodes. So the figure shows that the energy consumption slightly increases as the number of nodes increases. Since HHCC make use hop by hop congestion control, the overall energy consumption values are considerably less in HHCC when compared to the DCWCC protocol.

Fig. 6 presents the packet delivery Ratio (PDR) values for both the protocols. Due to the increased sending rate of the nodes, there is a slightly increase in the PDR values. But we can see that the PDR for HHCC increases, when compared to DCWCC protocol, as the sending rate is immediately controlled by the sources, when congestion occurs.

Fig.7 shows the throughput obtained for HHCC protocol and DCWCC protocol. It shows that the throughput of
HHCC is significantly more than the DCWCC, because of the above said reasons.

\section{CONCLUSION}

In this paper, we have proposed a Hop-by-hop congestion control protocol for wireless sensor networks to mitigate traffic contention. In our proposed protocol, each node calculates its node rank based on the parameters Buffer Size, Hop count, Channel Busy Ratio and MAC overhead. After estimating its rank, a node forwards this to its downstream node. When the node rank crosses a threshold $\mathrm{T}$, then the node will set the congestion bit in every packet it forwards. On receiving this node rank, the downstream node will first check for the congestion bit. If it is not set, it will simply computes its node rank and adds it to the rank obtained from its previous node and passes on to the next node. On the other hand, if the congestion bit is set, the node calculates the Rate Adjustment Feedback (RAF) based on the rank and transmits it towards the source as a feedback. On receiving this feedback, the source nodes will adjust their transmission rates and retransmit the data again. By simulation results, we have shown that our proposed protocol attains better throughput and packet delivery ratio with reduced energy consumption. In the future work, we will test our proposed protocol with larger networks with size 200 to 300 nodes with more number of sources.

\section{REFERENCES}

[1] Young-Duk Kim, Won-Seok Kang, Dong-Ha Lee, and Jae-Hwang Yu, "Distance Adaptive Contention Window Mechanism for Wireless Sensor Networks", in the proceedings of 23rd International Technical Conference on Circuits/Systems, Computers and Communications, pp 1693-1696, July 6-9, 2008.

[2] Md. Mamun-Or-Rashid and Choong Seon Hong, "Dynamic Contention Window based Congestion Control and Fair Event Detection in Wireless Sensor Network", In the Proceedings of 31st Korea information Processing Society (KIPS),Korea, pp. 1288-1290, May 2007.

[3] Costas Busch, Malik Magdon-Ismail, Fikret Sivrikaya, and Bulent Yener, "Contention-Free MAC protocols for Wireless Sensor Networks", In proceedings of the 18th International Conference on Distributed Computing (DISC 2004), Amsterdam, The Netherlands, pp. 245--259, October 2004.

[4] Huma Naushad, "A Survey on Energy-Efficient MAC Protocols for Wireless Sensor Networks", Mid Term Project Report Topics in Internet Research, Spring 2006.

[5] Md. Mamun-Or-Rashid, Muhammad Mahbub Alam, Md. Abdur Razzaque, and Choong Seon Hong, "Reliable Event Detection and Congestion Avoidance in Wireless Sensor Networks", SpringerVerlag Berlin Heidelberg 2007.

[6] Bret Hull, Kyle Jamieson, Hari Balakrishnan, "Mitigating Congestion in Wireless Sensor Networks", Proceedings of the 2nd international conference on Embedded networked sensor systems- 2004.

[7] Ian F. Akyildiz, Mehmet C. Vuran and Ozgur B. Akan "A CrossLayer Protocol for Wireless Sensor Networks", 40th Annual Conference on Information Sciences and Systems- 2006

[8] Wafa Ben Jaballah and Nabil Tabbane, "Multi path Multi SPEED Contention Window Adapter", IJCSNS International Journal of Computer Science and Network Security, VOL.9 No.2, February 2009.

[9] Hongwei Zhang, Anish Arora, Young-ri Choi and Mohamed G. Gouda, "Reliable Bursty Convergecast in Wireless Sensor Networks", Proceedings of the 6th ACM international symposium on Mobile ad hoc networking and computing- 2005.

[10] Ajit Warrier, Jeongki Min and Injong Rhee, "ZMAC: a Hybrid MAC for Wireless Sensor Networks", IEEE/ACM Transactions on Networking, June 2008.

[11] H. Zhai, X. Chen, and Y. Fang, "How well can the IEEE 802.11 wireless LAN support quality of service? IEEE Trans. Wireless Commun., vol. 4, no. 6, pp. 3084-3094, Nov. 2005. 
[12] Muhammad Mostafa Monowar, Md. Obaidur Rahman and Choong Seon Hong, "Multipath Congestion Control for Heterogeneous Traffic in Wireless Sensor Network", IEEE 10th International Conference on Advanced Communication Technology, Feb-2008

[13] Chonggang Wang, Kazem Sohraby, Victor Lawrence, Bo Li and Yueming $\mathrm{Hu}$, "Priority-based Congestion Control in Wireless Sensor Networks", Proceedings of the IEEE International Conference on Sensor Networks, Ubiquitous, and Trustworthy Computing-2006. (SUTC'06).

[14] Network Simulator: www.isi.edu/ns

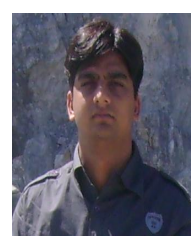

Kamal Kumar Sharma received B.Tech Degree in 2001 from S.K. Inst of Engineering and Technology, Kurukshetra university Kurukshetra in Electronics \& communication Engineering, M.Tech in 2007 from Punjab Technical University, Jalandhar in Electronics \& communication Engineering Department. He is doing Ph.D. in the field of wireless sensor networks from Punjab Technical University Jalandhar, INDIA. He is Associate Professor in Dept of Electronics and Communication Engg, R D Jindal group of colleges, Punjab. He has published 12 research papers in International/National Journals and Refereed International Conferences and two books in his core field.

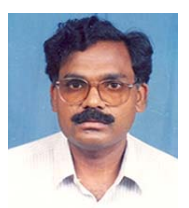

Dr. Ram Bahadur Patel received $\mathrm{PhD}$ from IIT Roorkee in Computer Science \& Engineering, PDF from Highest Institute of Education, Science \& Technology (HIEST), Athens, Greece, MS (Software Systems) from BITS Pilani and B. E. in Computer Engineering from M. M. M. Engineering College, Gorakhpur, UP. Dr. Patel is in teaching and Research \& Development since 1991. He has supervised several $\mathrm{M}$. Tech, and $\mathrm{M}$. Phil and $\mathrm{PhD}$ Thesis. He is currently supervising several $\mathrm{M}$. Tech, and $\mathrm{PhD}$ students. He has published more than 100 research papers in International/National Journals and Refereed International Conferences. Dr. Patel received several research awards. He is working as a professor in the department of Computer Engineering in MIT Deemed University Rajsthan INDIA.

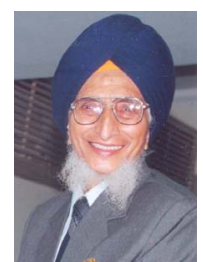

Dr. Harbhajan Singh is a engineering graduate of 1976 from Institutions of Engineers Calcutta INDIA in Electronics \& communication Engineering, and completed his Post graduate in Electronics Engineering from panjab Univeristy Chandigarh in 1983. Dr. Singh Completed his Ph.D. in 1987 in the field of ISDN and LAN. He is a Professor in Shree Sukhmani Institute of Engineering and Technology, Punjab. He has published more than 50 research papers in International/National Journals and Refereed International Conferences. 